\title{
PARTICIPATORY DESIGN OF A CONTINUOUS CARE ONTOLOGY Towards a User-Driven Ontology Engineering Methodology
}

\author{
Femke Ongenae $^{1}$, Lizzy Bleumers ${ }^{2}$, Nicky Sulmon ${ }^{3}$, Mathijs Verstraete ${ }^{3}$, Mieke Van Gils ${ }^{3}$, An \\ Jacobs $^{2}$, Saar De Zutter ${ }^{4}$, Piet Verhoeve ${ }^{4}$, Ann Ackaert ${ }^{1}$ and Filip De Turck ${ }^{1}$ \\ ${ }^{1}$ Information Technology Department (INTEC), Ghent University - IBBT, Gaston Crommenlaan 8, 9050 Ghent, Belgium \\ ${ }^{2}$ Research Centre for Studies on Media, Information and Telecommunication (SMIT), Brussels University (VUB) - IBBT, \\ Pleinlaan 2, 1050 Brussels, Belgium \\ ${ }^{3}$ Centre for User Experience Research (CUO), K.U. Leuven - IBBT, Parkstraat 45, bus 3605, 3000 Leuven, Belgium \\ ${ }^{4}$ Televic Healthcare NV, Leo Bekaertlaan 1, 8870 Izegem, Belgium \\ \{Femke.Ongenae, Ann.Ackaert, Filip.DeTurck\}@intec.ugent.be, \{Lizzy.Bleumers, An.Jacobs $\} @ v u b . a c . b e,\{$ Nicky.Sulmon, \\ Mieke.VanGils\}@soc.kuleuven.be, \{S.DeZutter,P.Verhoeve $\} @ t e l e v i c . c o m$
}

Keywords: Ontology engineering; Participatory; User-driven; Continuous care; eHealth.

\begin{abstract}
The patient room of the future would be able to sense the needs and preferences of the patients and nurses and adapt itself accordingly by combining all the heterogeneous data offered by the different technologies. This goal can be achieved by developing a context-aware framework, which exploits and integrates the heterogeneous data by utilizing a continuous care ontology. The existing ontology engineering methodologies are rather extreme in their choices to include domain experts. On the one hand, there are methodologies that only discuss the scope, use and requirements of the ontology with the domain experts. On the other hand, there are approaches in which the ontology is completely constructed by the domain experts by providing them with user-friendly and collaborative tools. In this paper, a participatory ontology engineering methodology is presented that finds a middle ground between these two extremes. The methodology actively involves social scientists, ontology engineers and stakeholders. The stakeholders participate in each step of the ontology life cycle without having to construct the ontology themselves or attribute a large amount of their time. The applicability of the methodology is illustrated by presenting the co-created continuous care ontology.
\end{abstract}

\section{INTRODUCTION}

\subsection{Background}

The ambient intelligent care room of the future will contain numerous devices that work together to support caregivers and residents in carrying out their everyday activities and tasks. The room will be able to sense the needs and preferences of the occupants and adapt itself accordingly by combining all the heterogeneous data offered by all the technology in the room such as sensors, wireless devices and input from caregivers given through multimodal interfaces, e.g., graphical user interfaces (GUIs) or speech recognition. However, nowadays the caregiver is responsible for the coordination, management and consultation of all these devices. The caregiver has to use several sources and devices to consult data and to keep it up to date even when carrying out a single task (Tentori et al., 2009). This is a very time consuming job.
Consider, for example, a patient, who suffers from a concussion, needs to be in a quiet and dark environment to heal. Today, it is the nurse who needs to configure the room to dim the lights each time he/she enters. Moreover, the nurse is responsible for alerting all the staff members and visitors that the room needs to be quiet and dark. If the nurse presses the wrong button or an uninformed person enters the room, this can cause physical pain for the patient. However, if the system would be aware of the patient's and nurse's needs, namely darkness for the patient and sufficient light for the nurse to work, the system can automatically turn on the light to the correct level when it detects that the nurse enters the room. Moreover, the system can display a message in the room or at the entrance of the room, reminding visitors and staff to keep the room dark and quiet.

The IBBT project ACCIO (Ambient aware provisioning of Continuous Care for Intra-muros Organizations) (Ongenae and et al., 2010) aims to tackle 
this problem by developing an intelligent, contextaware framework, which exploits and integrates the available heterogeneous data by utilizing an ontology (Gruber, 1993). An ontology formally describes the concepts in a domain, their attributes and their relationships. It can also contain classification rules, which are used to express the business logic of an application. This commonly agreed upon data-format can then be used to exchange the data and its attached domain model. In this way an ontology encourages re-use, communication, collaboration and integration.

Thus, the first step within the ACCIO project is the development of an ontology to support the continuous care of patients. This model has to contain information about the profile of the staff members and patients, which staff members are responsible for which tasks, administrative information, etc.

However, the incorporation of ontology engineering tasks in knowledge-empowered organizations such as hospitals can prove to be very difficult if not done in a way that is seamless to the day-to-day activities of the organization members (Kotis and Vouros, 2006). To resolve this issue, a participatory ontology engineering methodology is developed within the ACCIO project, which co-creates the ontology together with the stakeholders, i.e., nurses, caregivers, patients, doctors and professionals working for the healthcare industry. The methodology thus includes the stakeholders in every step of the design and development process.

\subsection{Related Work}

Although ontologies are widely accepted within the eHealth domain, most of these models focus on biomedical research, e.g., Galen Common Reference Model $^{1}$ or the Gene Ontology ${ }^{2}$. Little work has been done on the development of ontologies to support the continuous care of patients.

Well-known methodologies exist to construct an ontology such as Tove, Enterprise and Methontology (Pinto and Martins, 2004). However, these methodologies emphasize the role of the knowledge engineer. Domain experts are only involved during the specification phase. Moreover, no tools or methods are discussed to empower the domain experts to actively participate in the ontology life-cycle.

Recently, a human-centered approach (HCOME) (Kotis and Vouros, 2006) has been proposed where the active participation of domain experts is accentuated. This approach offers user-friendly and collaborative tools that allow the domain experts to construct

\footnotetext{
$1_{\text {http: //www.opengalen.org/index.html }}$

2 http://www.geneontology.org/
}

and discuss their own ontologies. The knowledge engineer only delivers (technical) support.

It can be noted that the current methodologies are rather extreme in their choice to involve domain experts in the ontology life-cycle. Within the ACCIO project, a methodology is developed that holds a middle ground between these two extremes. It promotes user participation while taking into account that time is a valuable resource within the eHealth domain. The methodology actively involves social scientists, ontology engineers and stakeholders, i.e., nurses, caregivers, patients, doctors and professionals working for the healthcare industry, in the ontology engineering process. Moreover, the methodology acknowledges that domain experts are no ontology engineers as such, or vice versa. The stakeholders participate in each step of the life cycle of the ontology without having to construct the ontology themselves or attribute a large amount of their time.

\subsection{Objective \& Paper Organization}

The goal of this paper is twofold. On one hand, the first steps towards a participatory ontology engineering methodology are described. On the other hand, the continuous care ontology, which resulted from applying the methodology, is presented.

The remainder of this paper is organized as follows. Section 2 describes the various user-driven methodologies and techniques used and evaluated for building an ontology. In Section 3, the developed continuous care ontology is discussed. Section 4 describes the lessons learned during the development of the methodology. Finally, the most important conclusions and future work are described in Section 5.

\section{ONTOLOGY CO-CREATION METHODOLOGY}

There are five widely accepted stages for building an ontology (Pinto and Martins, 2004):

- Specification: identify the scope and requirements

- Conceptualization: construct a conceptual model

- Formalization: translate the conceptual model into a formal model

- Implementation: implement the formal model in a knowledge representation language

- Maintenance: continuously evaluate and update the ontology to reflect changes in the domain

The following subsections describe the user-driven and participatory methodologies that were used and evaluated (Bleumers and et al., 2011) within the ACCIO project to achieve the goals of these stages. 


\subsection{Specification: Observations and Scenario Description}

To achieve a grounded, user-centered design process, the specification stage starts with creating a representative stakeholder group. Two major institutionalized continuous care settings were identified, namely residential (focus on care) and hospital care (focus on cure). A representative Flemish institution from each category closely collaborated in the project, namely Dominiek Savio Institute, which provides residential care to people suffering from cognitive and physical impairments, and OLV Hospital Aalst. Staff from other institutions and professionals working for the healthcare industry, e.g., Boone NV, a furnishing company, and Televic Healthcare NV, a nurse call system company, were also included in the stakeholder group.

Next, a hands-on workshop was organized in which the members of the project (i.e., the stakeholders most closely involved in the co-creation of the ontology), learned what ontology means and what ontology engineering entails, both from a social and a computer science perspective. A simple ontology was constructed through a series of exercises. Moreover, the workshop also aimed to generate discussion amongst the stakeholders on how a participatory ontology engineering methodology could be achieved. This is an essential step in interdisciplinary research and co-creation processes as the group diversity and the background knowledge is very different. A first essential step is learning to understand each other, so a better integration of concepts and views can be initiated in the subsequent development steps.

To get a clear picture of the scenarios in which an ontology-based, context-aware platform can optimize the provision of continuous care, observations were performed in the two collaborating institutions. The current daily practices were observed, e.g., helping a resident go to the toilet, giving medicine to a patient and communicating the status of a patient/resident with other staff members. Additionally, selected staff members, patients and residents were interviewed and logging data from the available systems, e.g., nurse call systems, were analyzed.

The observations were performed by the social scientists together with ontology engineers. All these observations were systematically represented in mind maps $^{3}$. An example is shown in Figure 1. These mind maps were also evaluated and validated with different stakeholders from the institutions, who were not necessarily involved in the observations.

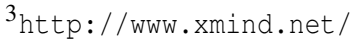

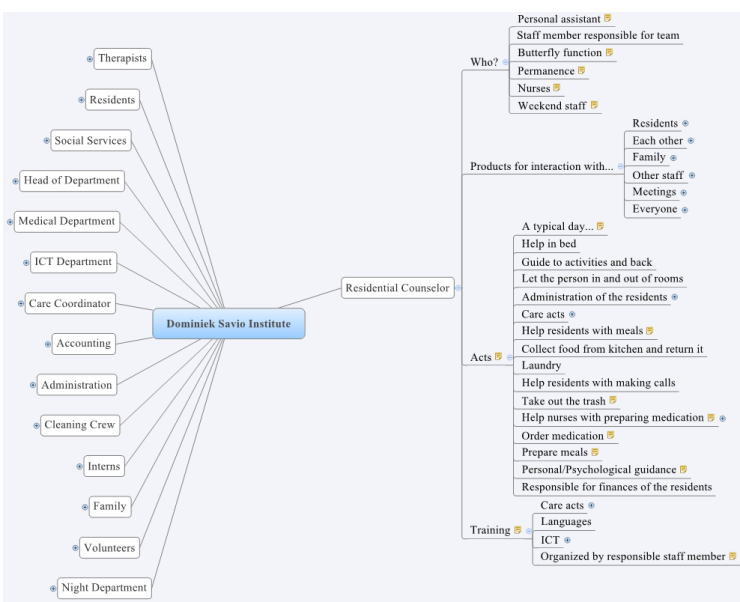

Figure 1: Example mind map, summarizing the observations at Dominiek Savio Institute.

By iterating these mind maps, a clear view of the current continuous care practices and two major areas for improvement were identified, namely information integration \& data provisioning at the point of care and an intelligent \& dynamic nurse call system. The first area can be summarized as providing the right information at the right time, at the right place for the right person. This requires an increased need for $\mathrm{mo}$ bile services to support data input, e.g.; care registration, and requested, e.g., medical data about patients, at the point of care. Moreover, information should be integrated, prioritized and filtered based on contextual information. Examples of this include only monitoring values that violate a threshold or only displaying recent information that pertains to the patient a staff member is currently caring for.

An intelligent nurse call system should use the integrated information about the staff and patients/residents to find the most appropriate staff member to handle a request of care from a patient/resident and at the same time optimize the current workflow in a $d y$ namic way. The current nurse call systems are mainly lacking the ability to adapt, to give feedback to the patient/resident and to give an overview of the current situation, e.g., localization of patients and staff.

To get a clearer view of all the information being exchanged during the continuous care practices, a document workflow was constructed. It outlines all documents (analog or digital) and formats of communication used during the care for patients/residents. The document workflow also describes the properties of the documents, such as the content, the specific aim, the author(s), the target audience, the storage location and the relationships to other documents.

As a final step in the specification stage, a sunnyday scenario was constructed. A scenario is a short story that describes the hypothetical use of a system 
to help develop a detailed and shared understanding of the context and activities of the users. In this case, the scenario describes the story of a resident of a residential care setting who becomes unwell and is transported to a hospital for treatment. Both the care for the resident in the residential care setting and the hospital are supported by the ontology-based, contextaware, continuous care system under development. The scenario is sunny-day because it is an ideal scenario in which the technology, e.g., the context-aware system, the sensors and actuators, optimally supports the needs of the users and the context, unconstrained by the current technological possibilities.

The scenario starts with the description of the various personas (Pruitt and Adlin, 2006). Personas are used to represent a particular user archetype of the software and include a name, a picture and demographic information. Personas communicate common attitudes, desires, behaviors and frustrations for a particular user group. Their main advantage is that they allow feeling real empathy for the user group, as they put a human face to a list of requirements. The scenario and accompanying personas, allowed the ontology engineers to keep in mind the various user groups whose information, requirements, activities and context should be represented in the ontology.

To summarize, the proposed user-driven specification stage consists of:

- Composing a representative stakeholder group

- Creating better knowledge of each other's field of expertise through a hands-on workshop comprising of a basic and comprehensive exercise

- Performing observations

- Analyzing and iterating the observations with the stakeholders, identifying areas where ICT can support and improve the current work processes

- Defining the scope and requirements of the ontology by creating a sunny-day scenario

- Iterating the sunny-day scenario with the stakeholders

\subsection{Conceptualization: Role-play Co-Design Workshop}

The two observed continuous care settings, namely Dominiek Savio Institute and OLV Hospital Aalst, are rather different, but the aim is to construct an ontology that can be used to build a context-aware platform, which supports and optimizes the continuous care practices in both settings. Moreover, the ontology should not only be applicable to the two specific settings studied during the observations. On one hand, the ontology should be abstract enough to be applied to all continuous care settings, while on the

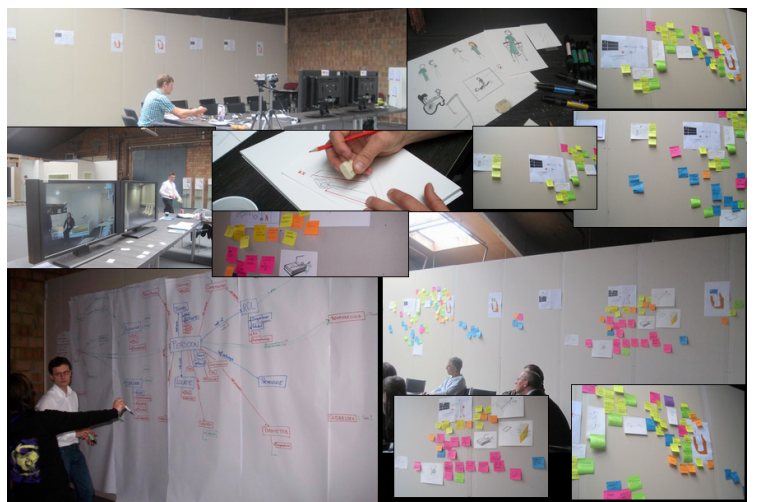

Figure 2: Role-play workshop at the PRoF1.0 room.

other hand it should remain specific enough to build practical applications on it. Therefore, it is important to identify the high-level concepts that are used with the same meaning within the continuous care domain and model them in a high-level ontology.

To achieve this goal a role-play co-design workshop was organized in the Patient Room of the Future (PRoF) 1.0 demo room ${ }^{4}$. Members from all stakeholder groups, e.g., caregivers and professionals working for the healthcare industry, participated in the workshop. The users were represented by both management and caregivers from different healthcare settings. A simplified, first version of the sunny-day scenario was visualized in a storyboard and used as central discussion point throughout the workshop, as can be seen in the top left corner of Figure 2. Participants took turns to play out the scenario in the PRoF1.0 room. Each participant received a persona card describing the character of the person they would be playing, e.g., an experienced nurse or an anxious patient. They also received a card describing the situational context of this specific scenario, e.g., patient has fallen out of bed during the night. The stakeholders not participating in the role-play, watched the scenario being played out on a TV outside the room and wrote down observations and comments, as visualized in the top left of Figure 2. After each role-play, a discussion was held amongst all the stakeholders. The discussion points were visualized on the storyboard by adding post-its and drawings of perceived problems, e.g., difficult for patient to alert a nurse, and opportunities, e.g., automatic fall detection, as visualized on the right of Figure 2.

Simultaneously, two ontology engineers followed the role-playing and discussions from the background, translating it to concepts and relations drawn as a graph on paper, as visualized in the bottom left corner of Figure 2. Intermittently, this high-level

\footnotetext{
${ }^{4}$ http://www.prof-projects.com/en/index.html
} 


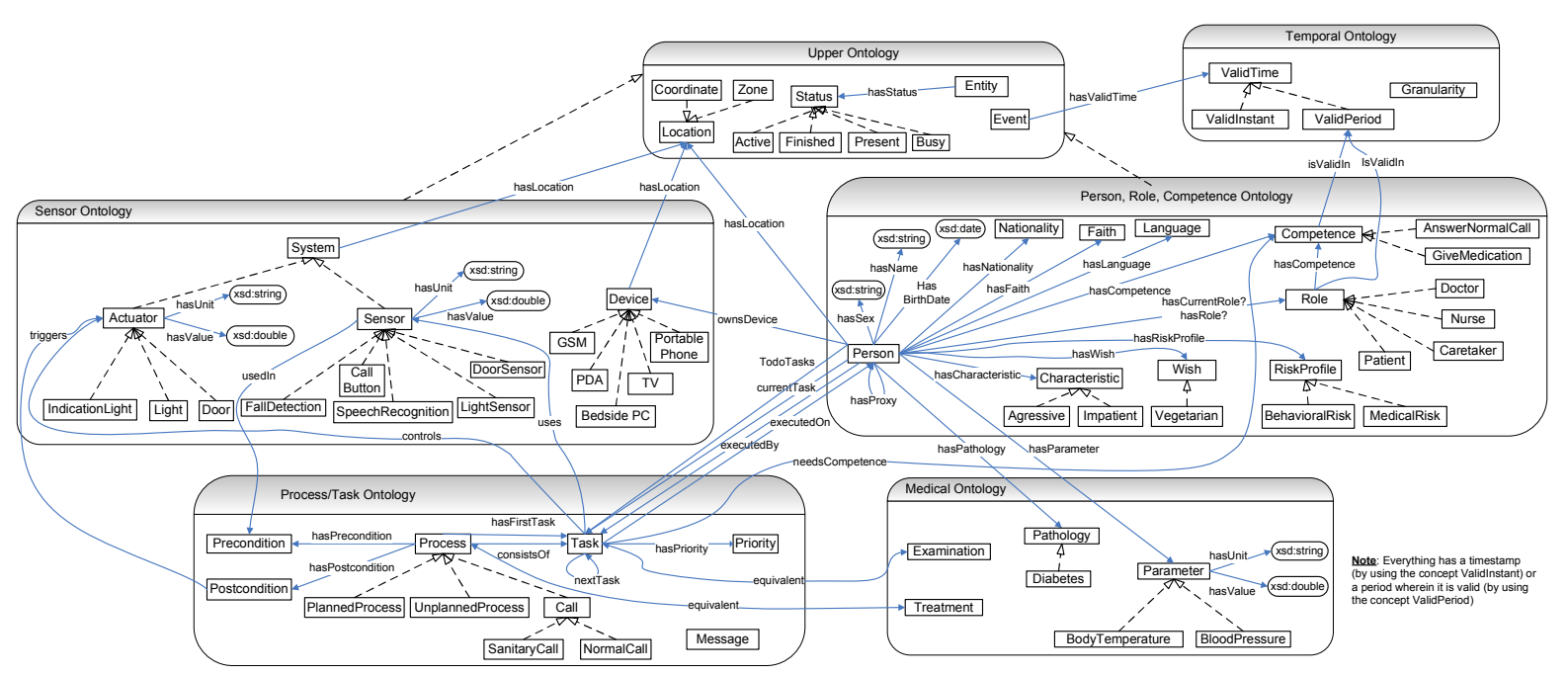

Figure 3: The high-level ontology resulting from the role-play co-design workshop at the PRoF1.0 demo room.

ontology was shown to the stakeholders for feedback.

The workshop resulted in a new iteration of the sunny-day scenario taking into account the feedback of the stakeholders and a first version of the high-level ontology, which is illustrated in Figure 3. As can be seen, 6 major sub-domains were identified, namely information pertaining to tasks \& processes, to person profiles, roles \& competences, to medical context, to sensor observations, to temporal information and to general upper concepts. The most important concepts within these sub-domains were identified (69 concepts in total). This allowed the ontology engineers to integrate the information obtained during the specification stage in this ontology, based on the mind maps and document workflow.

Based on this ontology, an investigation of existing ontologies was performed to evaluate if these could be re-used. The existing ontologies, which were found suitable, were imported. These included the Wireless Sensor Network (WSN) ontology (Verstichel and et al., 2010), the OWL-S Process onto$\log y^{5}$ and the SWRLTemporalOntology ${ }^{6}$.

As mentioned previously, the two settings under scrutiny during the observations are quite different. The major difference between the two is that residential care settings focus more on care, while hospitals focus more on curing the patients. Therefore, it was decided to conceptualize two low-level ontologies, containing concepts specific to these settings. These low-level concepts are always subconcepts of concepts in the high-level ontology.

The low-level ontologies were constructed by analyzing the document workflows and mind maps and

\footnotetext{
5 http://www.w3.org/Submission/OWL-S/

$6_{\text {http: }} / /$ protege.cim3.net/cgi-bin/wiki.pl? SWRLTemporalontology
}

extracting concepts such as roles, competences, tasks (care acts) and profiles. The two resulting low-level ontologies were compared to identify common concepts used within both settings with the same meaning. These concepts were moved to the high-level ontology. The resulting ontologies are discussed in Section 3.

To summarize, the proposed user-driven conceptualization stage consists of:

- Performing one or more role-playing workshops based on the sunny-day scenario to define the high-level concepts for the ontology, making sure all stakeholders are represented in the workshop

- Mapping the high-level ontology on existing, reusable ontologies

- Extracting low-level concepts by studying the results from the observations, propagating concepts used in all settings to the high-level ontology

- Updating the sunny-day scenario with the feedback of the stakeholders obtained during the roleplaying workshops

\subsection{Formalization: Decision-Tree Co-Design Workshop}

In this stage, the conceptual description developed in the previous stage is transformed into a formal model. This means that the concepts are defined through $a x$ ioms that restrict the possible interpretations for the meaning of those concepts, e.g., defining which competences a certain role has, which competences are needed to perform a task or which sensor observations are valid. A lot of these restrictions were derived from the data extracted during the observations or the sunny-day scenario discussions. However, there were still some gaps in the ontology pertaining to the 


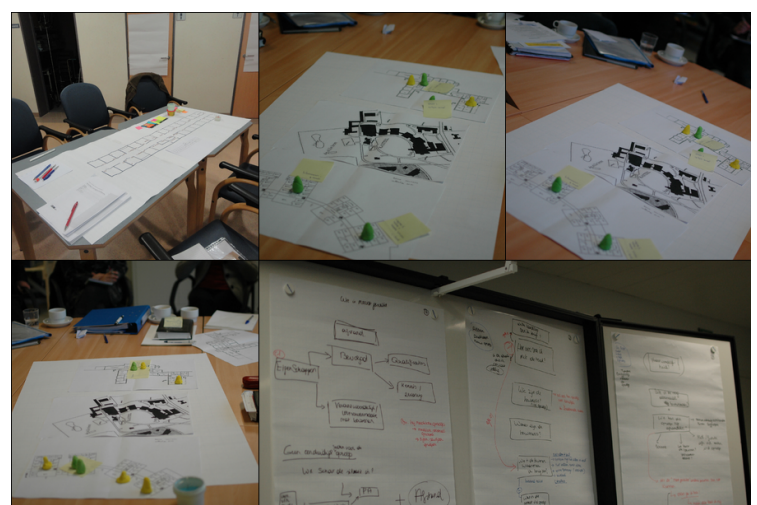

Figure 4: Decision-tree workshop: scenario is visualized on a layout and high-level concepts, derived from the requested information, are summarized in a decision tree.

way care requests or nurse calls are prioritized and assigned to caregivers.

These gaps were filled by organizing decision-tree workshops. The goal of these workshops was to capture the decision process that the caregivers propose or find ideal to prioritize and assign care requests or nurse calls in a decision tree. This decision tree can then be translated to axioms in the ontology. To make sure the context-dependent factors are captured, the workshop was organized twice: once with stakeholders from the residential care domain and once with stakeholders from the hospital.

At the start of the workshop, the participants described a complex situation involving care requests or nurse calls. Next, the participants were asked to suppose they were an intelligent system that had a complete overview of the care setting and the current situation. The task of this system is to find the most appropriate staff member to handle a care request from a patient/resident. A few of the complex situations described by the participants were selected to further discuss how this intelligent system should ideally handle them, i.e., prioritize and assign them. The situations were illustrated on a layout of the institution. The participants, playing the role of the system, could collect additional information about the situation by asking questions, e.g., How many staff members are present in the department? What are their roles? Who made the request? Instead of immediately giving an answer, a discussion was first held about the importance of the requested information, e.g., Why do you feel the answer to this question is pertinent? Does everyone agree? Can you give examples of answers to this question? Finally, the question was answered and the answer was visualized on the layout, as can be seen in Figure 4.

The asked questions gave the ontology engineers insights into which information is important to make a

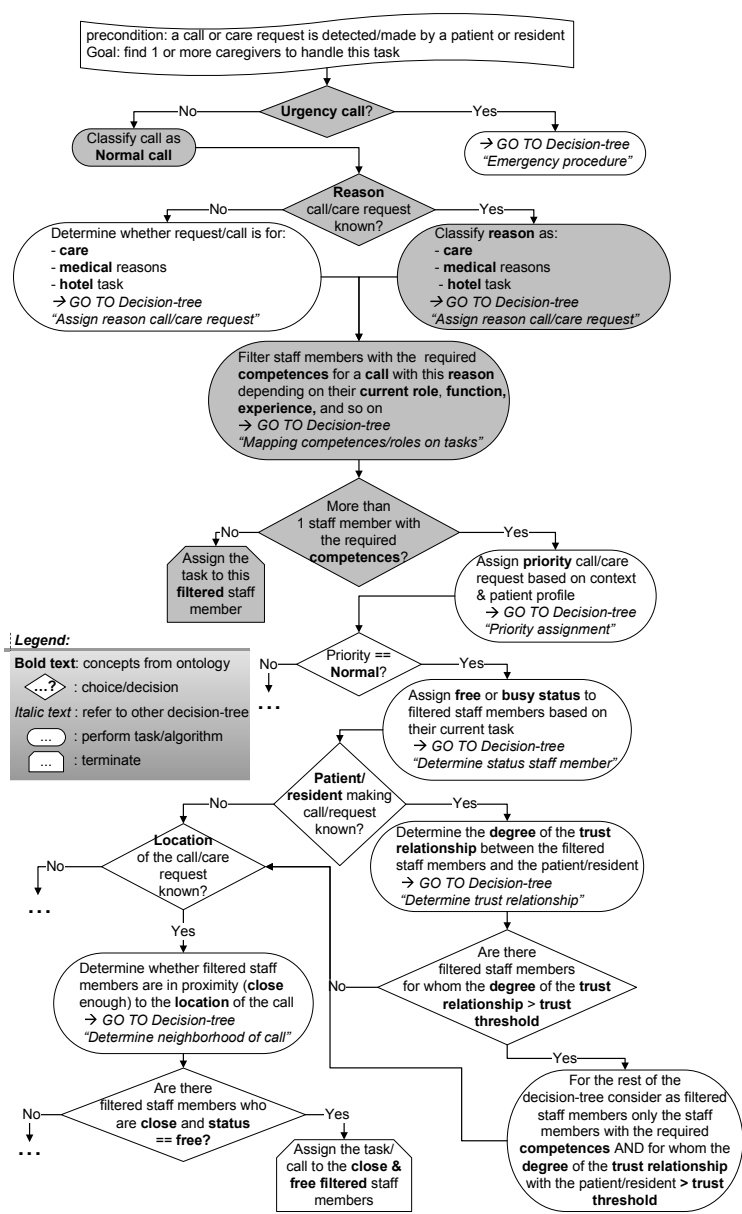

Figure 5: Decision tree that finds the appropriate staff member to handle a call or care request.

decision. Moreover, the order in which the questions are asked gave insight into the importance of this information for making the decision. During the workshop, this information is visualized on paper in the form of a decision tree, as shown in the bottom right corner of Figure 4. This decision tree thus contains the high-level concepts to which the questions of the participants pertained, e.g., location or task priority.

After the workshop, these results were transformed to more formal decision trees for each setting. After all the workshops, the decision trees were compared to extract common parts. The common parts deliver input for the high-level ontology, while the other branches are encoded in the low-level ontologies. Finally, the decision trees are translated to axioms and rules in the implementation stage (see Section 2.4).

As an example, Figure 5 shows a part of the common decision tree that finds the most appropriate staff members to handle a call or care request based on the data in the ontology. Words indicated in bold refer to concepts from the high-level ontology. The algo- 
rithm first determines whether the call is an urgency call. Each care setting has its own procedure for handling these highest priority calls. These procedures are encoded in separate, context-dependent decision trees to which this decision tree refers. If the call is normal, the decision tree determines whether the reason for the call is medical, care or a "hotel" task, e.g., a request for a glass of water. Based on this classification, staff members are sought with the required competences for carrying out such tasks. If there are multiple staff members available with the required competences, the decision tree assigns the most appropriate staff members to the call based on their current task, location, trust relationship with the patient and the priority of the call.

To summarize, the proposed user-driven formalization stage consists of:

- Extracting axioms and rules from the observations

- Performing one or more decision-tree workshops with a carefully selected group of stakeholders, each workshop has a very specific goal to fill a specific formal gap in the ontology

- Updating the sunny-day scenario with the feedback obtained during the decision-tree workshops

\subsection{Implementation \& Maintenance}

In the implementation stage, the conceptual graphs and decision trees are translated into an ontology language. As the goal is not to overburden the stakeholders, they are not actively involved in this stage. However, to prevent losing sight of the user requirements when making implementation decisions, the ontology engineers constantly validate the ontology under development with the sunny-day scenario.

The Protégé editor ${ }^{7}$ was used to develop the continuous care ontology in the Ontology Web Language $(O W L)^{8}$. The Pellet Reasoner ${ }^{9}$ was used to check the consistency and the classification of the ontology. The $S W R L$ rule language ${ }^{10}$ was used to express rules using concepts from the ontology. The resulting continuous care ontology is discussed in Section 3.

The maintenance stage consists of continuously evaluating, updating and correcting the ontology. This work is ongoing within the ACCIO project. The developed ontology is currently being evaluated with the stakeholders by organizing additional role-play workshops in the PRoF1.0 demo room. These workshops also include stakeholders who were not involved in the other stages to make sure the ontology is

\footnotetext{
${ }^{7}$ http://protege.stanford.edu/

8 http://www.w3.org/TR/owl-features/

${ }^{9}$ http://pellet.owldl.com/

10 http://wWw.w3.org/Submission/SWRL/
}

widely applicable across the continuous care domain.

Meanwhile, the intelligent, context-aware framework is being developed and installed in the PRoF1.0 room. The aim is to show how intelligent applications can be built on top of this ontology. As a proof of concept an intelligent nurse call system (Ongenae and et al., 2011) is being built which finds the most appropriate staff member to handle a call made by a patient/resident by using all the information collected in the ontology. This nurse call system makes use of all the sensor data which can be collected in the PRoF1.0 demo room, e.g., light sensors and intelligent bed able to sense the vital parameters of a patient/resident. The nurse call system is also able to adjust the status of the devices in the room based on the context captured in the ontology, e.g., turn on the light at a certain level when a staff member enters the room or adjust the temperature based on the presence of people in the room and their preferences.

Once this intelligent nurse call system is installed, stakeholders will be invited to the PRoF1.0 demo room to play with the system, criticize, discuss and evaluate it. This evaluation will give input about the correctness and applicability of the ontology.

\section{THE CONTINUOUS CARE ONTOLOGY}

As mentioned previously, the main difference between residential care settings and hospitals is that the first focus more on care, while the latter focus more on curing the patients. Consequently, the ontology was split into a high-level ontology, modelling the concepts that are used with the same meaning within the continuous care domain, and two low-level ontologies, one focusing on care and the other on cure. These low-level ontologies contain concepts that are used with a different meaning in both settings or that are used within residential care, but never within hospitals and vice versa. The following paragraphs give a concise overview of the high-level ontology.

Sensor Ontology: This ontology models the information delivered by the numerous sensors and actuators present in the ambient intelligent care room of the future. The most important concepts are visualized in Figure 6. The concepts preceded with the wsn prefix are imported from the Wireless Sensor Network (WSN) ontology, which was developed within the IBBT project DEUS ${ }^{11}$ and allows attaching meaning to the data values monitored by sensors.

\footnotetext{
$11_{\text {http: //www }}$ ibbt.be/en/projects/ overview-projects/p/detail/deus
} 


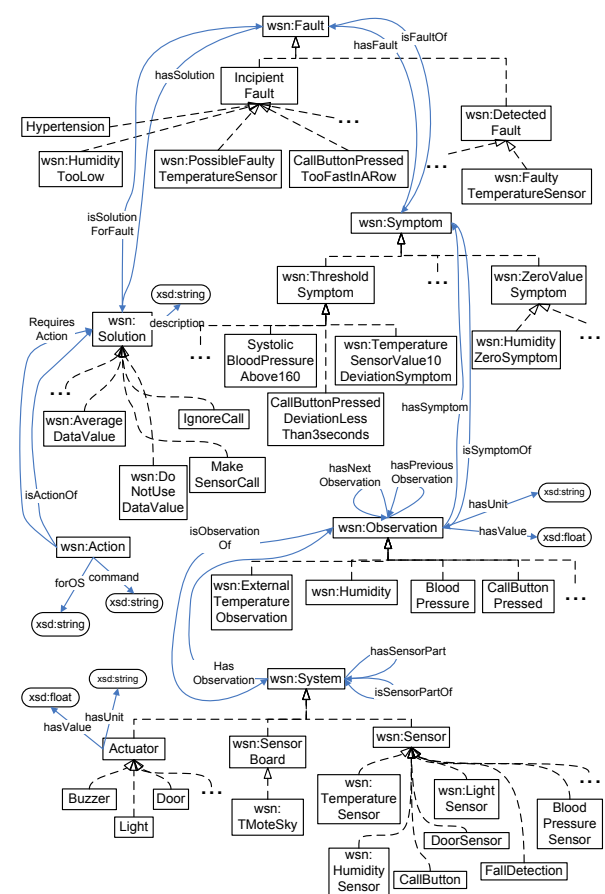

Figure 6: The most important concepts of the imported WSN and the Accio Sensor ontology.

The System concept models a system and its components, e.g., sensors. An Observation represents a data value monitored by a system. Rules added to the ontology allow detecting specific phenomena, modeled as Symptom concepts, in these observations, e.g., temperature observation deviates more than 10 degrees from the last. Axioms reclassify these symptoms as Fault concepts, e.g., the temperature sensor is possibly faulty, and even as a Solution, e.g., do not use that temperature value. This WSN ontology was extended with systems, sensors and actuators, e.g., nurse call buttons or fall detection systems, and their associated observations, faults and solutions that play an important role in continuous care settings. Some examples are shown in Figure 6.

Task \& Process Ontology: This ontology models continuous care process workflows. The most important concepts are shown in Figure 7. The classes preceded by the owls prefix are imported from the $O W L$ $S$ Process ontology 5 . The Process concept models a process that can return some new information and produces a change in the environment based on the information it is given and the context. This is described by the hasInput, hasOutput, hasPrecondition and haseffect relations. A process can be composed of several other processes through the CompositeProcess and ControlConstruct concepts. The Accio Task ontology extends this ontology by introducing the Task concept, which is a subclass of Process and

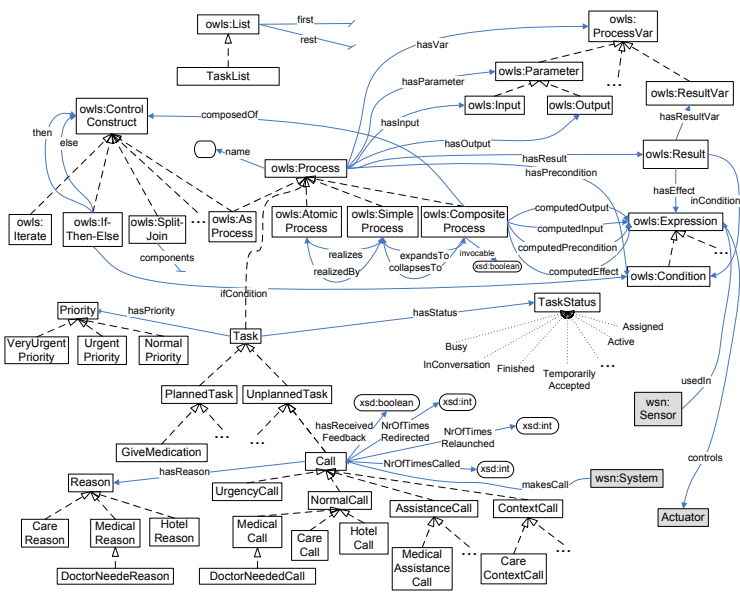

Figure 7: The most important concepts of the imported OWL-S Process and the Accio Task ontology.

is further divided into planned and unplanned tasks. Some examples of each are shown at the bottom of Figure 7. Each task has an associated Status, e.g., Assigned or Finished, and Priority. From the observations and workshops it was clear that the stakeholders preferred three levels of priority, namely very urgent, urgent and normal. This Task concept is used to model the continuous care tasks. Consider, for example, the task of assigning a person to a call, for which the decision tree is shown in Figure 5. A Call is modeled as an unplanned task. The various possible types of calls, e.g., urgency or normal, and the possible reasons for the call, i.e., medical, care and hotel, are also modeled. For each type of call, its preconditions, e.g., a patient pushes a button, its input, e.g., the patient, its output, i.e., the assigned staff members, and its effects, e.g., the staff members' portable phones ring, are modeled by using concepts from the OWL-S Process ontology.

Profile Ontology: This ontology models the profile information about staff members and patients/residents that was indicated by the stakeholders in the different co-design workshops as important. The most important concepts are visualized at the bottom of Figure 8. Each Person is associated with a Profile, which consists of a basic and a risk profile. The basic profile models the biological, psychological and sociological information. Some examples are shown in Figure 8. This information needs to be inputted into the system or extracted from documents, e.g., patient's medical file. The risk profile is defined by classification axioms and rules. This allows a reasoner to automatically obtain the risk profile of the patient by reasoning on the information in the basic profile. Finally, the Profile ontology also contains concepts and rules to model the trust relationship between two people, e.g., it models that each doctor has a therapeutic 
relationship of high degree with his/her patients.

Role \& Competence Ontology: This ontology, of which the most important concepts are shown in the top part of Figure 8, defines each role that can occur in a continuous care setting by its competences through classification axioms, e.g., the Doctor Role is defined as a role which has all the medical competences. This allows writing algorithms that find the most appropriate staff members to fulfill a task based on the required competences. Each person is associated with competences and roles through five relationships. hasfunction indicates the primary role of a person, i.e., the role for which this person was primarily hired. hasRole models all the roles a person can have, e.g., the head of the department who is also a nurse. The role a person is currently fulfilling within the care setting is represented by hascurrentRole. Finally, hasDiplomaCompetence and hasExperienceCompetence model extra competences a person has acquired by following courses, e.g., a caregiver who is trained to perform some medical tasks, or through experience.

Context Ontology: This model represents the contextual environment information. The most prevalent concepts are shown in the middle of Figure 8. The most important concept is the Troop, which is a logical grouping of entities that belong together, e.g., a patient with all his/her devices, sensors, actuators, room, bed and equipment. The composition of a troop dynamically changes based on the context, e.g., the location or status of the people, devices and equipment. This is defined through rules.

Medical Ontology: To model the medical knowledge, the Galen Common Reference Model ${ }^{1}$ was imported and connected with the concepts from the Accio ontology as illustrated in Figure 8. For example, the Pathology concept is connected with the Person concept from the Accio Profile ontology.

Temporal Ontology: It also important to model time in the ontology, e.g., tasks that need to be performed before a certain point in time or work processes that are adjusted according to the time of day. For this the SWRLTemporalOntology ${ }^{6}$ was imported. This ontology defines a temporal model that can be used to model complex interval-based temporal information. It also defines a library of SWRL built-ins to perform temporal reasoning. The Accio Temporal ontology extends this ontology to model the current temporal context, e.g., the current season or shift.

SWRL rules: On top of this ontology rule-based algorithms are developed that contain the algorithms to optimize and automate continuous care. These rules infer new knowledge based on the data available in the ontology. Efficient and fast notifications made

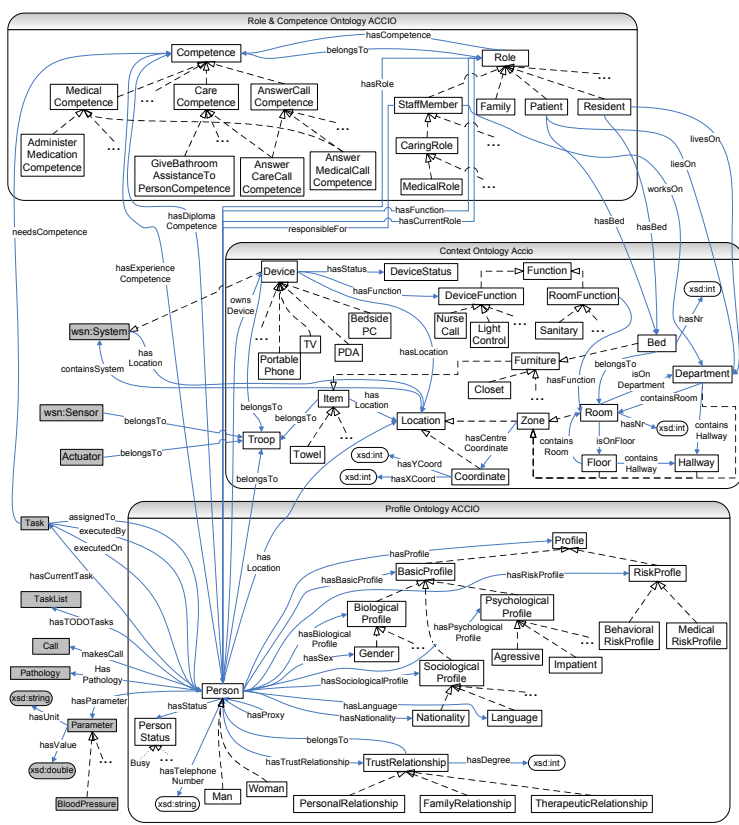

Figure 8: The most important concepts of the Profile, Context and Role \& Competence Ontology.

by these algorithms allow appropriate actions to be taken by the staff. An example of a work process that can be optimized this way is the assignment of caregivers to calls or care requests for which the decision tree is shown in Figure 5. For example, the grey path in Figure 5 is encoded as a SWRL Rule ${ }^{10}$ as follows:

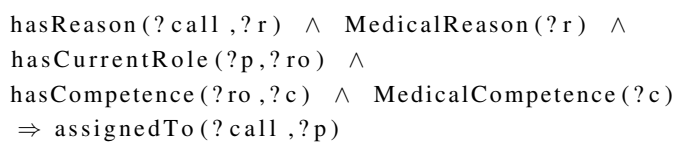

\section{Lessons Learned}

In this section, the most important lessons learned during the co-creation of the ontology with the participatory methodology are discussed. More information can be found in Bleumers, et al. (2011).

Timing: One of the goals was to achieve user participation beyond collecting the requirements of the ontology in the specification phase, but without actually pushing them in the role of ontology engineers and thus overburdening them. The stakeholders are mainly involved through the workshops and observations. The observations comprised of 1 week in each continuous care setting, during which staff members were followed and interviewed. The workshops lasted 2 to 3 hours on average. The workshops were distributed amongst the available participants, while maintaining the group of each workshop representative of the different stakeholders in the domain. Par- 
ticipants often remarked that the sessions seemed too short to get to the bottom of the issue at hand. However, it was difficult to lengthen the workshops as participants often could not make themselves available that long. In line with a suggestion of one of the participants, a follow-up session was coupled with each workshop. This allows presenting the output of the workshop and the achieved results. This permits to briefly discuss pressing issues not handled during the workshop, to evaluate the output and to illustrate to the stakeholders that their input was taken into account and that is was time well spent. It was also concluded that while the described methodology limits the time that has to be invested by the stakeholders, it does require a large amount of time and effort from the social scientists and ontology engineers.

Elicitate out-of-the-box thinking: During the workshops, it was noted that it was difficult for stakeholders to look beyond their current situation and work practices. This issue was resolved by explicitly triggering participants to think out-of-the-box, e.g., by taking them out of their usual role and practices with the persona and situation cards in the role-play workshops.

Connecting ontology engineers and stakeholders: One of the challenges during the workshops was the facilitation of the communication between the ontology engineers and stakeholders. It became apparent that bridges needed to be build between them. This was done in various ways. First, the ontology engineers took part in the observations to get an idea of the current work practices of the stakeholders. Second, bridges were also used during the workshops, e.g., the storyboard in the role-play workshop or the decision-tree. Finally, the resulting ontology and axioms were communicated with the stakeholders in an easily understandable format, e.g., document workflows, mind maps, graphs and decision-trees.

Learning by doing: A hands-on approach was used during the workshops, e.g., exercises in the ontology workshop, role-playing in the role-play workshop and question-and-answer process in the decision-tree workshop. Participants were also stimulated to reflect on sometimes higly complex issues. It was found that participants much appreciated this approach of action and reflection. It allowed them to reflect on their current practices, enhanced their understanding of the topic and elicitated discussion.

\section{CONCLUSIONS}

In this paper a participatory ontology engineering methodology is proposed which promotes user parti- cipation while taking into account that time is a valuable resource. The methodology actively involves ontology engineers, social scientists and stakeholders, i.e., nurses, caregivers, patients, doctors and professionals working for the healthcare industry, in the ontology engineering process. The user-driven methodologies and techniques to achieve this participatory ontology engineering methodology were presented in detail and validated by building a continuous care ontology. Future work will focus on developing and validating user-driven techniques to support the maintenance stage of the ontology life-cycle.

\section{ACKNOWLEDGEMENTS}

Part of this research was supported by the IBBT Project ACCIO. F. Ongenae would like to thank the IWT for her Ph.D. grant. The role-play workshop was organized in the PRoF1.0 demo room in Poperinge, Belgium $^{4}$. We thank all the participants in ACCIO for their valuable contribution to the project.

\section{REFERENCES}

Bleumers, L. and et al. (2011). Towards ontology cocreation in institutionalized care settings. In Proc. of Pervasive Health 2011, Dublin, Ireland. Piscataway, USA: IEEE.

Gruber, T. (1993). A translation approach to portable ontology specifications. Knowledge Acquisition, 5:199220.

Kotis, K. and Vouros, G. (2006). Human-centered ontology engineering: the hcome methodology. Knowledge and Information Systems, 10(1):109-131.

Ongenae, F. and et al. (2010). User-driven design of an ontology-based ambient-aware continuous care platform. In Proc. of Pervasive Health 2010, Munich, Germany. Piscataway, USA: IEEE.

Ongenae, F. and et al. (2011). An ontology-based nurse call management system (oncs) with probabilistic priority assessment. BMC Health Services Research, 11(26).

Pinto, H. and Martins, J. (2004). Ontologies: how can they be built? Knowledge and Information Systems, 6(4):441-464.

Pruitt, J. and Adlin, T. (2006). The persona lifecycle: keeping people in mind throughout product design. Morgan Kaufmann, San Mateo, USA.

Tentori, M., Segura, D., and Favela, J. (2009). Mobile Health Solutions for Biomedical Applications, chapter VIII: Monitoring hospital patients using ambient displays. Medical Information Science Reference, USA.

Verstichel, S. and et al. (2010). Distributed ontology-based monitoring on the ibbt wilab.t infrastructure. In Proc. of TridentCom 2010, Berlin, Germany. Berlin/Heidelberg, Germany: Springer. 